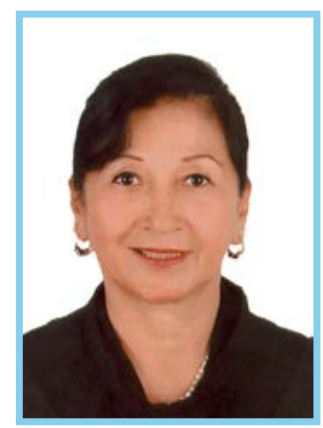

Mg. María Peralta Lino

\section{APROXIMACIONES A UN MODELO DE FORMACIÓN PROFESIONAL}

\section{RESUMEN}

El artículo describe el interés por el modelo del proceso formativo, como expresión del pensamiento y proyección de las comunidades académicas, en el sentido y contenido del marco científico que le da sentido y valor. Refiere posiciones teóricas que validan la importancia de la visión aproximativa del modelo que orienta la progresiva elaboración del saber en la práctica intercultural de la docencia en la Universidad. Incluye referencias a Santa Magdalena Sofía Barat acerca del rol orientador del modelo pedagógico en la formación de valores intelectuales y capacidades cognitivas, con lo cual se reafirma el impacto del modelo en la cultura de las instituciones educativas en contextos de complejidad. Finalmente, moviliza líneas de acción derivadas de la trascendencia de la visión como fuerza orientadora del modelo de formación profesional que, según Gimeno Sacristán (1988), es la representación conceptual, simbólica, indirecta, esquemática, parcial, selectiva de aspectos de esa realidad.

\section{ABSTRACT}

The present article describes the interest for the learning process model as a thought expression and academic community's projection, in the meaning and content of the scientific framework that provides meaning and value. Relates theoretical positions that validate the importance of the approximate model vi sion that guides the progressive development of knowledge in the intercultural practice of the teaching at the University. Include referents to Santa Magdalena Sofia Barat about the Pedagogical Model Guiding role in the formation of intellectual values and cognitive abilities, which confirm the impact on the culture of educational institutions in contexts of complexity. Finally, mobilizes action lines derived from the importance of vision as a guiding force of the model in professional training, which, according to Gimeno Sacristán (1988), is the conceptual, symbolic, indirect, schematic, partial, selective representation of that reality.

\section{PALABRAS CLAVE}

Modelo educativo, proceso formativo, capacidades cognitivas, modelo de formación profesional.

\section{KEYWORDS}

Educative model, informative process, cognitive capacities, model of professional training.
$1 \begin{aligned} & \text { lo largo del tiempo y en } \\ & \text { el escenario académico, } \\ & \text { Lha sido una constante la }\end{aligned}$ temática referida a la dinámica profesional que caracteriza la tarea de los formadores en la Universidad. Al respecto, se considera necesario fortalecer el sentido y contenido del marco científico que orienta el saber profesional y la práctica docente en las instituciones de educación superior desde determinadas categorías y criterios.

La experiencia profesional, en la complejidad de estos contextos, me permite afirmar que el interés por la calidad del contenido temático, la profundidad de la interrogante, la perspectiva de la investigación y los medios al servicio del aprendizaje constituyen hoy en día, la expresión más reiterada y visible del interés por la naturaleza y calidad del pensamiento y del saber en las instituciones de Educación Superior donde la actividad principal es la formación profesional. 
El participar en esta plática y ser parte de la búsqueda incesante de respuestas y certezas, me permite arribar en algunas ideas y conclusiones. En primer lugar, pienso que efectivamente, se trata de un saber pensar valioso y privilegiado, muy propio del académico comprometido e identificado con la actividad intelectual de la Universidad.

Indudablemente, es una actividad caracterizada por la fuerte pero flexible articulación entre saber y saber hacer. Esta conexión tendría que ser intencional favoreciendo la reflexión y la acción. Los docentes en la universidad necesitan orientar su saber pensar al saber actuar desde un referente de valor. Un saber pensar que, desde esta perspectiva, se entendería como el principio que orienta el diseño, desarrollo y evaluación, de un modelo de formación profesional.

Entonces, es evidente que esta dinámica formativa que incluye políticas, programas y experiencias académicas, supone la presencia de un modelo que de una y otra forma orienta, valora y condiciona los procesos y sus resultados. Un modelo de formación sin lugar a dudas, subyace en la actuación y en la dinámica del claustro universitario y ocurre a partir de las competencias y convicciones; del pensamiento libre y creativo; del compromiso con el principio que representa la visión y la misión más allá de la simple adecuación al formato o a las reglas.

Es tan importante esta aseveración que Hebe Tizio (2005), advierte que mientras la regla intenta dar medida a la acción para que se ajuste a ella o para que las acciones resulten conformes y adecuadas; el principio que en este caso refiere el saber pensar, señala un punto de partida natural e inconfundible y una base común que da suficiente margen al proyecto del modelo.

Esta referencia al principio y a la regla, marcaría la distinción entre el orden y el concierto invariante; entre el estándar y la orientación esencial de una práctica ya no en función de la regla sino más bien, en razón de la calidad del pensamiento y del saber. De esta manera, se contribuye a esclarecer, en algo, el sentido e intención de la temática centrada en el interés por un modelo que definitivamente representa el sentido y el valor del saber y de la práctica profesional.

En este ámbito académico donde, cada vez se hacen más frecuentes las prácticas orientadas a concretar el pensamiento de los docentes y de los estudiantes, resulta imprescindible definir la función del formador en relación al propósito y contenido del proyecto educativo que, de existir, haría visible al modelo.

Escudero (1981), define el modelo como la construcción que representa, de manera simplificada, una realidad o fenómeno con la finalidad de delimitar algunas de sus dimensiones o variables. El autor añade que esta representación permite una visión aproximativa, a veces intuitiva, que orienta estrategias de investigación para la verificación de la relación entre variables con intención de aporte a la progresiva elaboración del saber.

Para Gimeno Sacristán (1988), un modelo representa la realidad y supone, a la vez, un distanciamiento de la misma. Refiere el autor, que el modelo es una representación conceptual, simbólica, indirecta, esquemática, parcial, selectiva de aspectos de esa realidad. Afirma, que lo que pretende el modelo es configurar y estructurar una práctica formativa basada en una teoría (parte teórica) y en una práctica (aplicación del modelo) de manera abierta, adaptable y modificable.

De acuerdo a lo expresado, los modelos explican cómo la actuación, en este caso, de los docentes universitarios, estaría condicionada por su pensamiento, el cual como ya se ha mencionado, se entiende como una construcción personal ante la enseñanza y el aprendizaje. Por ello, también, es fundamental hacer referencia a la 
importancia de incluir y valorar las expectativas y necesidades de los docentes. Estos al estar en constante interacción, ejercen una dinámica intencional que representa la construcción y la práctica intercultural del pensamiento y, por lo tanto, la concreción de lo ideal y valioso en la formación.

En este punto, es vital hacer referencia al maravilloso contenido del documento La Interioridad en la Pedagogía de Sofía Barat (1997) elaborado por la Hna. Josefina Escartín Gaspar, expertaenla vidayescritos dela Santa, presentado en el Encuentro Interprovincial de Educación celebrado en Santa María de Huerta, Soria (España). En el capítulo II "Base Firme de Valores Intelectuales", la autora escribe que "Magdalena Sofía había experimentado la fuerza educadora de los valores intelectuales y quiso ponerlos en la base del Plan de Estudios, como cimiento sólido de la personalidad de los alumnos. Conocía, también, que la INTERIORIDAD se fundamenta en ellos y que su proceso se desarrolla a la vez que las capacidades cognitivas. Por esto, su objetivo prioritario fue ENSENAAR A PENSAR para forjar personas con pensamiento propio, capaces de crítica, dispuestas a comprometerse en la construcción de una sociedad nueva" ${ }^{1}$

Al citar, en este artículo, tan valiosa y singular referencia es preciso apreciar su impacto en la calidad de los modelos de formación. Especialmente, en su función de facilitar la comprensión e interpretación correcta del hecho educativo y su orientación formativa en el contexto de la universidad donde saber y saber pensar es fundamental.

Definitivamente, la misión formadora motiva al educador $y$, en este caso, al formador, a desarrollar sus procesos desde un modelo que le permite identificar y analizar el marco referencial en el que actúa para establecer el alcance y los límites de su pensamiento y de su propia acción. Se estima que así encuentra, de manera natural, la forma de introducir en su actuación lógicas más pertinentes ante situaciones de gran complejidad.

En este sentido, y sea cual fuese la orientación del modelo, este debe representar la posibilidad de afiliación y ubicación de los sujetos en una estructura de relaciones a partir de la reflexión y de una propuesta de contenidos valiosos como centro de la acción formadora. La respuesta, indudablemente, serán las filiaciones auténticas al modelo considerando su particularidad y la forma de inscribirse en la cultura y en los modos de representación social.

Al tener en cuenta esta consideración, se incluiría en el modelo la intencionalidad de la investigación - acción y de la formación de los maestros para la comprensión. Conviene aquí citar a Lawrence Stenhouse, quien estima que al ser la enseñanza un arte y el currículo construido por docentes un valor, es necesario enfatizar que la formación y el desarrollo profesional solo ocurren en el referente de una sólida preparación académica y del rigor de la investigación sobre la propia práctica para modificarla y mejorarla.

Así, en la dinámica de este contexto y tal como ya se ha mencionado, un valor fundamental del modelo también serán los contenidos del saber profesional que signifiquen una apuesta por todos aquellos sujetos que debido a la exigencia y complejidad del proceso tienen riesgo de quedar en una situación de exclusión. Por ello, será necesario mediar didácticamente con mayor dedicación el acceso a la cultura global y compleja del mundo actual, sentando las bases de un pleno y sensible escenario de desarrollo humano.

Medina Rivilla (2006), afirma que el saber, a partir de esta referencia intercultural, se construye desde el conocimiento intenso y

1 Escartín, Josefina, rscj. (1997). La Interioridad en la Pedagogía de Sofía Barat. p. 31. 
justificado de las concepciones personales, creencias, habilidades y actitudes. Asimismo, afirma que significan un insumo muy interesante los problemas de la ciencia y las síntesis interdisciplinarias de los diversos modos de vivir y entender el mundo de cada cultura en su interdependencia y complejidad.

Finalmente y con la intención de proponer, desde estas líneas, un espacio de encuentros, intereses y coincidencias en relación al saber pensar y al modelo en la formación profesional, es de interés hacer referencia al rol metodológico que implica la construcción y búsqueda de interrogantes y respuestas que indagan sobre cómo realizar el despliegue de este modelo de formación.

\section{REFERENCIAS}

Paquay, Leopold; Altel, Marguerite y Perrenoud, Phillipe (2005). La Formación Profesional del Maestro. Estrategias y Competencias. México D. F., México: Fondo de Cultura Económica.

Alemany Arrebola, Inmaculada; Jiménez Jiménez, María Ángeles y Sánchez Fernández, Sebastián (Coords.) (2012). Formación del Profesorado para la Diversidad Cultural. Madrid, España: La Muralla S. A.

Ministerio de Educación, Dirección General de Educación
Como afirma la Hna. Josefina Escartín, rscj., "enseñar a pensar supone un itinerario de formación intelectual al que hay que dedicar atención, sabiduría y tiempo, para que sea maduro y coherente". Por lo tanto, será necesario contar con educadores y formadores vocacionados, estudiosos y comprometidos con su obra, constituyéndose en el equipo que trabaja solidariamente para que la acción formadora de cada uno intensifique la de los demás. Pero también, será importante contar con referentes de valor como el modelo para clarificar los trayectos que contribuyan de manera efectiva y eficaz a las transformaciones socioculturales de nuestra sociedad.
Intercultural Bilingüe y Rural, Dirección General de Educación Superior y Técnico - Profesional, Dirección de Educación Superior Pedagógica (2012). Diseño Curricular Experimental para la Formación de docentes en las Carreras de Educación Inicial Intercultural Bilingüey Educación Primaria Intercultural Bilingüe. Lima, Perú: Autor.

Yuri, José Alberto (Compilador) (2009). La Formación Docente. Complejidad y Ausencias. Buenos Aires, Argentina: Encuentro.

\section{Unıfé}

